\title{
Hydration structure and dynamics of the favipiravir antiviral drug: \\ A molecular modelling approach.
}

\author{
Ioannis Skarmoutsos ${ }^{1, *}$, Guillaume Maurin ${ }^{2}$, Elvira Guardia ${ }^{3}$, Jannis Samios ${ }^{4}$ \\ ${ }^{1}$ Theoretical and Physical Chemistry Institute, National Hellenic Research Foundation, \\ Vas. Constantinou 48, GR-116 35, Athens, Greece \\ ${ }^{2}$ ICGM, Univ. Montpellier, CNRS, ENSCM, Montpellier, France \\ ${ }^{3}$ Departament de Física, Universitat Politècnica de Catalunya, Campus Nord-Edifici B4-B5, Jordi Girona \\ 1-3, Barcelona E-08034, Spain. \\ ${ }^{4}$ Department of Chemistry, Laboratory of Physical Chemistry, National \& Kapodistrian University of \\ Athens, Panepistimiopolis 157-71, Athens, Greece
}

\begin{abstract}
The hydration structure of the Favipiravir antiviral drug, at infinite dilution in water, was investigated by employing a systematic molecular modelling approach. An effective interaction potential model was employed for Favipiravir, using the intramolecular geometry and charge distribution from quantum chemical calculations performed in the present treatment and adopting well-established Lennard-Jones parameters from the literature. The hydration structure and related dynamics were further investigated by means of classical molecular dynamics simulations. These calculations have revealed the existence of different types of hydrogen bonds between Favipiravir and the surrounding water molecules, with continuous lifetimes in the sub picosecond range and intermittent lifetimes in the range of 0.8-5.4 ps. The self-diffusion coefficient of Favipiravir of $0.58 \cdot 10^{-9} \mathrm{~m}^{2} \cdot \mathrm{s}^{-1}$ at $298.15 \mathrm{~K}$ was found to be three times lower than the value obtained for water in solution, while comparable to the to the values measured for other common painkillers and antiinflammatory drugs (e.g. paracetamol, aspirin, ketoprofen), antibiotics (e.g. tetracycline, trimethoprim, Penicillin G) and corticosteroids for asthma treatment (beclomethasone, prednisone). It was also revealed that the rotational motions of Favipiravir are more retarded in comparison with water and this is reflected on the calculated reorientational correlation times of specific intramolecular vectors. The results obtained in the present study could be useful in pharmaceutical applications, such as pharmacokinetics and computer-aided docking studies to evaluate the efficiency of this particular antiviral drug.

*Corresponding Author. Email: iskarmoutsos@hotmail.com
\end{abstract}




\section{Introduction}

Despite the enormous progresses in pharmacy and medicine, particularly over the last two decades, viral infections continue to be a significant threat to public health with very significant societal and economic impacts ${ }^{1,2}$. For this reason, the scientific community is constantly seeking for novel approaches to develop innovative drugs and new therapeutics ${ }^{3}$. However, only a few antiviral treatments have proven to be effective and there is a critical need to discover therapeutic approaches for a wide range of diseases. Especially nowadays, the current circumstances, with the emerging viral infections (COVID-19) for which effective drugs or vaccine therapies are not available, urgently call for the development of antiviral drugs with enhanced functionality ${ }^{4,5}$ and an in-depth understanding of the molecular mechanisms that drive their action.

From a physicochemical perspective, structure-based drug discovery methods ${ }^{6,7}$ can be employed to develop novel antiviral drugs. However, in urgent situations like nowadays, taking into account that the development of novel small-molecule drugs could take a very long time, virtual high-throughput screening of existing drugs with regulatory approval and potential for therapeutic use could provide very fast a list of potential candidates ${ }^{8}$ for further experimental testing. At the same time, a deeper understanding of the solvation mechanisms and the diffusivity of active pharmaceutical ingredients in water and aqueous solutions could be correlated with their in vivo drug dissolution rate, as it has been suggested in the literature ${ }^{9,10}$. Water is the primary component of the human body, and providing insight of the diffusion mechanisms of drugs in aqueous solutions could be crucial for a deeper understanding of drug permeability phenomena in biological membranes. The presence of an aqueous diffusion layer, or unstirred water layer, adjacent to the intestinal membrane has long been regarded as a potential barrier for intestinal absorption of pharmaceutical compounds ${ }^{11-13}$. Therefore, understanding and providing quantitative description of the diffusion mechanisms of active pharmaceutical ingredients in water could shed light on the role of unstirred water layers and interface transitions in passive transport through permeation barriers. Such a fundamental knowledge can be used as a first-step selection approach to classify drugs based on their bioavailability, which is strongly correlated to their physicochemical properties such as the solubility and diffusivity 
in water ${ }^{9,10,14}$. Since the use of advanced experimental techniques to measure accurately the diffusivity of drug is quite complex and time consuming, molecular modelling ${ }^{15,16}$ appears as a good alternative to provide fast and reliable estimation of this data.

Since the predictive capability of molecular simulation techniques in computational pharmaceutics ${ }^{17}$, either by molecular dynamics or Monte Carlo, strongly relies on the quality of the potential model to describe the intermolecular interactions of the systems, several physicochemical parameters need to be carefully taken into account when developing these models. Particularly in the case of the prediction of transport properties of drugs, either in solution or in biological membranes, a crucial factor which strongly affects the diffusivity of the drug molecules is their polarity ${ }^{18}$. High level quantum mechanical calculations can be very helpful in estimating the polarity of drug molecules. These calculations, when used in combination with techniques which estimate the atomic charge distributions by fitting procedures which reproduce the molecular electrostatic potential at a number of points around the molecule, can provide accurate descriptions of the polarity and the corresponding Coulombic interactions as implemented in force fields used to model the behavior of drug molecules in condensed phases ${ }^{19}$.

The main aim of the present work was first to develop an effective interaction potential model for the Favipiravir antiviral drug molecule, by employing high level $a b$ initio quantum chemical calculations to obtain the intramolecular geometry and charge distribution and using well-established Lennard-Jones parameters from the literature. And then, using this potential model, to perform classical molecular dynamics simulations in order to predict the hydration structure and related dynamics, as well as the diffusivity of this particular molecule. Favipiravir (T705, 6-fluoro-3-hydroxypyrazine-2-carboxamide) is a pyrazine analog, slightly soluble in water, that has demonstrated potent antiviral activity against a broad spectrum of viruses ${ }^{20-23}$. Favipiravir is a selective and potent inhibitor of influenza viral RNA polymerase and a promising drug for the treatment of infections caused not only by the influenza virus but also by a wide range of RNA viruses, including the Ebola virus ${ }^{20,22,23}$. Favipiravir has been recently suggested as one of the potential inhibitors interfering with the RNA-dependent RNA polymerase of the SARSCoV-2 coronavirus ${ }^{5}$. Chinese authorities have also recently granted approval to use 
Favipiravir in clinical trials to see whether it works against SARS-CoV-2 as well. In view of the importance of the use of Favipiravir, the development of a model which could provide important information regarding the behavior of this drug in aqueous solutions becomes indispensable. Apart from that, such a model could be used in further future molecular docking simulation studies to understand in detail the molecular-scale phenomena which could help in targeting the infection pathways of several viruses, with particular emphasis on the SARS-CoV-2 coronavirus.

\section{Computational Details}

Quantum chemical calculations were first employed to geometry optimize Favipiravir. These calculations were performed using Gaussian $09^{24}$ at the MP2 level of theory, which in general is efficient to recover dispersion effects, using the cc-pVTZ ${ }^{25}$ basis set. Frequency analysis was also carried out for the optimized structure ensuring the presence of no imaginary modes. The calculated structural parameters, such as bond lengths and angles of this planar molecule were found to be in good agreement with available experimental crystallographic data ${ }^{26}$, extracted from the data reported in a previous theoretical study ${ }^{27}$, as it can be seen in Table 1.

The atomic charge distribution in Favipiravir was calculated by employing the CHELPG scheme $^{28}$, to reproduce the molecular electrostatic potential at a number of points around the investigated optimized structure. In order to build a potential model, also called force field, for classical MD studies, these atomic charges were used in combination with existing sigma $(\sigma)$ and epsilon ( $(\varepsilon)$ 12-6 Lennard-Jones (LJ) parameters taken from the OPLS-AA force field ${ }^{29}$. The intramolecular geometry was kept rigid in this particular model. These LJ parameters have been parametrized for many different classes of organic molecules ${ }^{29}$, in order to accurately predict the thermodynamic, structural and dynamics properties of organic fluids in a wide range of thermodynamic conditions. A summary of the intramolecular coordinates, charges and LJ parameters corresponding to each type of atoms of the Favipiravir molecule are presented in Table 2. The optimized structure of Favipiravir and the names corresponding to the different atom types in the force field are also presented in Figure 1. 
Table 1: Calculated bond lengths and angles of Favipiravir, presented together with available experimental crystallographic data

\begin{tabular}{|c|c|c|c|c|c|}
\hline $\begin{array}{c}\text { Bond } \\
\text { Lengths }(\AA)\end{array}$ & Calculated & $\begin{array}{c}\text { Experimental } \\
\text { (Ref. 26) }\end{array}$ & $\begin{array}{c}\text { Bond Angles } \\
\text { (deg) }\end{array}$ & Calculated & $\begin{array}{c}\text { Experimental } \\
\text { (Ref. 26) }\end{array}$ \\
\hline $\mathrm{O}_{\mathrm{h}}-\mathrm{H}_{\mathrm{h}}$ & 0.992 & 0.820 & $\mathrm{H}_{\mathrm{h}}-\mathrm{O}_{\mathrm{h}}-\mathrm{C}_{2}$ & 105.05 & 109.5 \\
\hline $\mathrm{C}_{\mathrm{C}}-\mathrm{O}_{\mathrm{C}}$ & 1.238 & 1.244 & $\mathrm{O}_{\mathrm{h}}-\mathrm{C}_{2}-\mathrm{C}_{3}$ & 122.35 & 123.5 \\
\hline $\mathrm{O}_{\mathrm{h}}-\mathrm{C}_{2}$ & 1.328 & 1.328 & $\mathrm{C}_{3}-\mathrm{C}_{\mathrm{C}}-\mathrm{O}_{\mathrm{C}}$ & 120.95 & 119.7 \\
\hline $\mathrm{C}_{\mathrm{C}}-\mathrm{C}_{3}$ & 1.487 & 1.481 & $\mathrm{C}_{3}-\mathrm{C}_{\mathrm{C}}-\mathrm{N}_{\mathrm{am}}$ & 115.02 & 117.3 \\
\hline $\mathrm{C}_{\mathrm{C}}-\mathrm{N}_{\mathrm{am}}$ & 1.341 & 1.318 & $\mathrm{C}_{\mathrm{C}}-\mathrm{N}_{\mathrm{am}}-\mathrm{H}_{\mathrm{N}}$ & $119.04,119.23$ & $119.1,120.0$ \\
\hline $\mathrm{H}_{\mathrm{N}}-\mathrm{N}_{\mathrm{am}}$ & $1.004,1.005$ & 0.860 & $\mathrm{C}_{2}-\mathrm{C}_{3}-\mathrm{C}_{\mathrm{C}}$ & 120.20 & 120.7 \\
\hline $\mathrm{C}_{2}-\mathrm{C}_{3}$ & 1.410 & 1.397 & $\mathrm{O}_{\mathrm{C}}-\mathrm{N}_{\mathrm{am}}-\mathrm{H}_{\mathrm{N}}$ & 124.02 & 123.1 \\
\hline $\mathrm{C}_{1}-\mathrm{C}_{2}$ & 1.394 & 1.390 & $\mathrm{C}_{\mathrm{C}}-\mathrm{C}_{3}-\mathrm{N}_{2}$ & 118.00 & 118.0 \\
\hline $\mathrm{C}_{2}-\mathrm{N}_{3}$ & 1.337 & 1.340 & $\mathrm{C}_{2}-\mathrm{C}_{3}-\mathrm{N}_{2}$ & 121.80 & 121.4 \\
\hline $\mathrm{C}_{1}-\mathrm{N}_{3}$ & 1.331 & 1.306 & $\mathrm{C}_{3}-\mathrm{C}_{2}-\mathrm{N}_{3}$ & 120.74 & 120.8 \\
\hline $\mathrm{C}_{1}-\mathrm{H}_{\mathrm{R}}$ & 1.081 & 0.930 & $\mathrm{C}_{3}-\mathrm{N}_{2}-\mathrm{C}_{4}$ & 115.95 & 116.3 \\
\hline $\mathrm{C}_{4}-\mathrm{F}$ & 1.332 & 1.339 & $\mathrm{~N}_{2}-\mathrm{C}_{4}-\mathrm{F}$ & 117.22 & 116.8 \\
\hline $\mathrm{C}_{4}-\mathrm{N}_{2}$ & 1.311 & 1.295 & $\mathrm{C}_{1}-\mathrm{C}_{4}-\mathrm{F}$ & 119.42 & 119.9 \\
\hline $\mathrm{C}_{3}-\mathrm{N}_{2}$ & 1.342 & 1.335 & $\mathrm{~N}_{3}-\mathrm{C}_{1}-\mathrm{H}_{\mathrm{R}}$ & 118.16 & 119.4 \\
\hline
\end{tabular}


Table 2: Intramolecular coordinates, LJ parameters and charges corresponding to each atom type of the force field employed in the MD simulations to model Favipiravir.

\begin{tabular}{|c|c|c|c|c|c|c|}
\hline $\begin{array}{c}\text { Atom } \\
\text { Type }\end{array}$ & $\mathbf{x}(\mathbf{\AA})$ & $\mathbf{y}(\mathbf{\AA})$ & $\mathbf{z}(\AA)$ & $\boldsymbol{\sigma}(\boldsymbol{\AA})$ & $\boldsymbol{\varepsilon}(\mathbf{k c a l} / \mathbf{m o l})$ & $\mathbf{q}(|\mathbf{e}| \mathbf{~}$ \\
\hline $\mathrm{C}_{1}$ & 2.079878 & 0.874058 & 0.000005 & 3.55 & 0.070 & 0.035 \\
\hline $\mathrm{C}_{2}$ & -0.163666 & 1.262621 & 0.000002 & 3.55 & 0.070 & 0.608 \\
\hline $\mathrm{C}_{3}$ & -0.389370 & -0.129326 & -0.000015 & 3.55 & 0.070 & -0.113 \\
\hline $\mathrm{C}_{4}$ & 1.830084 & -0.496904 & -0.000013 & 3.55 & 0.070 & 0.458 \\
\hline $\mathrm{H}_{\mathrm{R}}$ & 3.092810 & 1.252692 & 0.000019 & 2.42 & 0.030 & 0.147 \\
\hline $\mathrm{C}_{\mathrm{C}}$ & -1.777820 & -0.661919 & -0.000047 & 3.75 & 0.105 & 0.688 \\
\hline $\mathrm{N}_{\mathrm{am}}$ & -1.872179 & -1.999190 & 0.000020 & 3.25 & 0.170 & -0.753 \\
\hline $\mathrm{H}_{\mathrm{N}}$ & -2.780506 & -2.426465 & 0.000136 & - & - & 0.363 \\
\hline $\mathrm{H}_{\mathrm{N}}$ & -1.029773 & -2.547839 & 0.000129 & - & - & 0.363 \\
\hline $\mathrm{O}_{\mathrm{C}}$ & -2.752681 & 0.101357 & -0.000022 & 2.96 & 0.210 & -0.614 \\
\hline $\mathrm{N}_{2}$ & 0.623281 & -1.010033 & -0.000019 & 3.25 & 0.170 & -0.337 \\
\hline $\mathrm{N}_{3}$ & 1.080491 & 1.753448 & 0.000010 & 3.25 & 0.170 & -0.429 \\
\hline $\mathrm{F}$ & 2.853843 & -1.348308 & 0.000002 & 2.85 & 0.061 & -0.255 \\
\hline $\mathrm{O}_{\mathrm{h}}$ & -1.157059 & 2.143395 & 0.000021 & 3.07 & 0.170 & -0.591 \\
\hline $\mathrm{H}_{\mathrm{h}}$ & -1.984994 & 1.597612 & 0.000044 & - & - & 0.430 \\
\hline
\end{tabular}

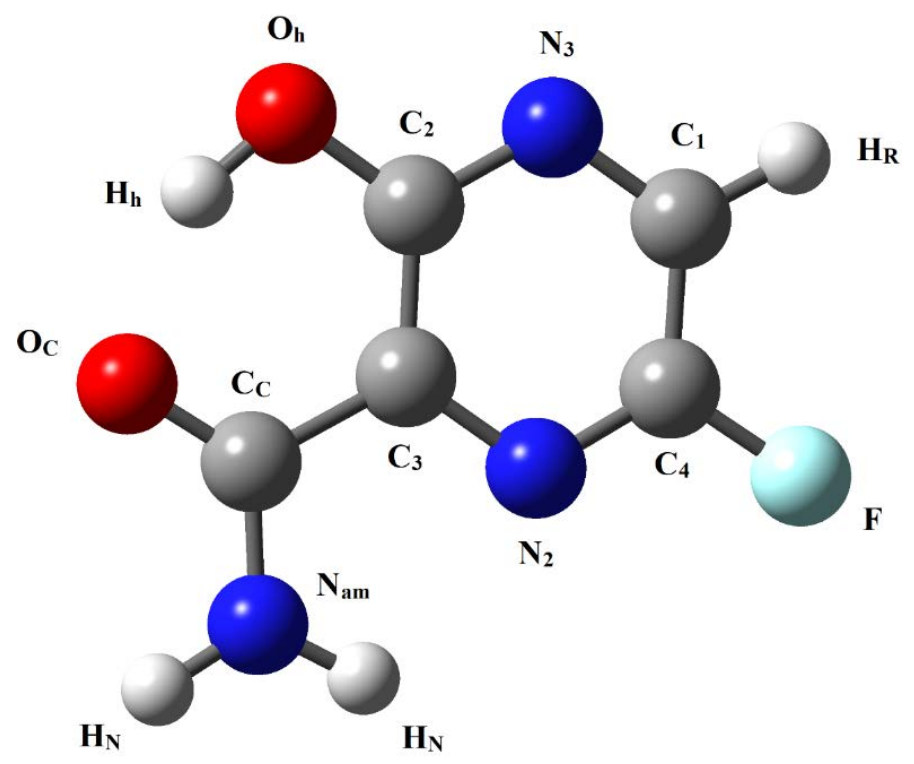

Figure 1: Optimized intramolecular structure of Favipiravir at the quantum chemical level and the names corresponding to the different atom types in the force field. 
Note that the molecular dipole moment of Favipiravir for this particular model is $3.53 \mathrm{D}$. The development of force fields for water has been a topic of constant interest over the last decades and a very large variety of force fields have been proposed to describe the properties of water ${ }^{30,31}$. In the present study we have used the TIP4P/2005 potential model $^{32}$, which provides a realistic description of the properties and anomalies of water in a wide range of thermodynamic conditions.

The solvation structure of Favipiravir at infinite dilution in pure ambient water $(\mathrm{T}=298.15$ $\mathrm{K}$ and $\mathrm{P}=1$ bar) has been subsequently investigated via classical $\mathrm{MD}$ simulations. one Favipiravir molecule was placed among 499 water molecules inside a cubic simulation box with dimensions corresponding to the bulk water density. The initial configuration of the simulated system was prepared by using the Packmol ${ }^{33}$ software and the system was then equilibrated by performing an initial 2 ns MD simulation at the isothermal-isobaric (NPT) ensemble. The density of the equilibrated system was then calculated by a subsequent 2 ns production NPT-MD run. Using the box dimension corresponding to the calculated density of the system at 1 bar, the system was then re-equilibrated at the canonical (NVT) ensemble for 2 ns and finally, the system properties under equilibrium were evaluated in a subsequent 2 ns NVT-MD production run. The equations of motion were integrated using a leapfrog-type Verlet algorithm ${ }^{16}$ with an integration time step of $1 \mathrm{fs}$. The temperature was fixed to $298.15 \mathrm{~K}$ and the pressure to 1 bar by coupling the system to a Nose-Hoover thermostat $^{34}$ and barostat ${ }^{35}$ with relaxation times of 0.5 ps, respectively. The rigid body equations of motion for the Favipiravir and the water molecules were expressed in the quaternion formalism. A cut-off radius of $12.0 \AA$ has been applied for all Lennard-Jones interactions and long-range corrections have also been taken into account. To account for the long-range electrostatic interactions the standard Ewald summation technique has been used. The simulation runs were performed using the DL_POLY ${ }^{36}$ simulation code.

\section{Results and Discussion}

The hydration structure of Favipiravir in ambient liquid water was investigated in terms of the atom-atom and center of mass - center of mass (COM-COM) radial distribution functions (RDF) g (r). The most representative RDFs are presented in Figure 2. From the shape of the calculated atom-atom RDF it can be clearly seen that the Favipiravir atoms 
which exhibit the strongest interactions with the water molecules are the hydrogen atoms $\mathrm{H}_{\mathrm{N}}$ of the $-\mathrm{NH}_{2}$ group, the oxygen atom of the carbonyl group $\mathrm{O}_{\mathrm{C}}$ and the oxygen atom of the hydroxyl group $\mathrm{O}_{\mathrm{h}}$. The $\mathrm{RDF}$ for the $\mathrm{H}_{\mathrm{N}}-\mathrm{O}_{\mathrm{w}}$ pair exhibits a first, low-intensity, peak at $2.03 \AA$, followed by a minimum at $2.53 \AA$. Similarly, the $\mathrm{O}_{C}-\mathrm{H}_{\mathrm{W}} \mathrm{RDF}$ exhibits a first peak at $1.78 \AA$, followed by a minimum at $2.43 \AA$. The corresponding position of the first maximum and minimum for the $\mathrm{O}_{\mathrm{h}}-\mathrm{H}_{\mathrm{W}} \mathrm{RDF}$ are 1.93 and $2.38 \AA$, respectively. The fact that these maxima and minima are located in very short intermolecular distances are clear indications of the existence of hydrogen bonding (HB) between Favipiravir and the water molecules. However, the low intensities of these corresponding peaks signify that these interactions are not very strong.

The shape of the RDF for the $\mathrm{F}-\mathrm{H}_{\mathrm{w}}$ pair, with an even lower intensity shoulder, located in the range 1.93-2.43 $\AA$, also indicates the existence of weak short-range HB interactions between the water hydrogen atoms and the fluorine atom of Favipiravir. As it can be observed, depending on the Favipiravir atom which interacts with water, the water molecules can act either as HB donors or acceptors. When the water molecules interact with the $\mathrm{H}_{\mathrm{N}}$ or the $\mathrm{F}$ atoms of Favipiravir they act as $\mathrm{HB}$ acceptors, interacting with their oxygen atoms. On the contrary when they interact with the $\mathrm{O}_{\mathrm{c}}$ and $\mathrm{O}_{\mathrm{h}}$ atoms, they act as HB donors, interacting with their hydrogen atoms. The size of the first solvation shell of Favipiravir can also be estimated by the shape of the calculated COM-COM RDF. As it may be observed the COM-COM RDF exhibits a first maximum at $5.43 \AA$, followed by a minimum at $6.48 \AA$, which corresponds to the first solvation shell radius. The coordination number corresponding to the first solvation shell of Favipiravir is 33.6. 

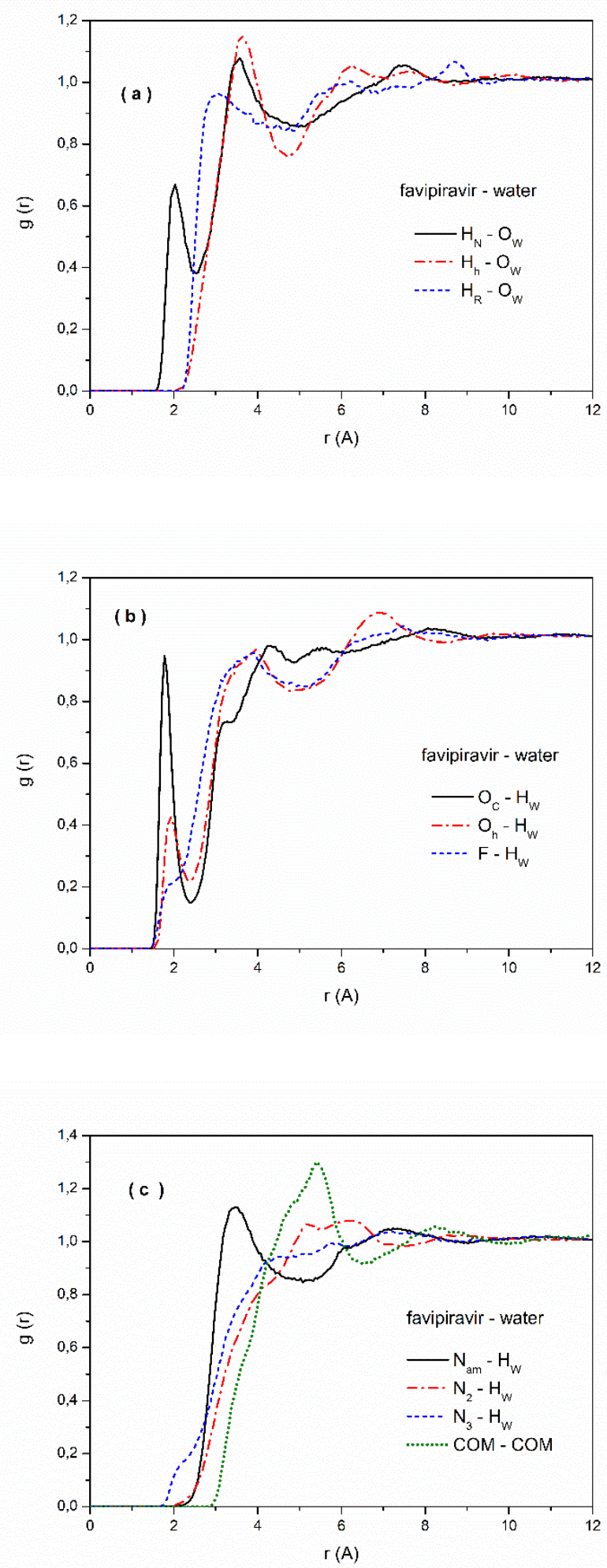

Figure 2: Calculated atom-atom and COM-COM radial distribution functions, corresponding to the Favipiravir-water pairs. 
In order to provide a more quantitative description of the hydrogen bonds formed between the Favipiravir molecule and its closest water neighbor molecules, a HB analysis was performed by employing common geometric criteria. According to these criteria, a hydrogen bond between a Favipiravir atom X and a hydrogen or oxygen atom of water (depending if water acts as HB acceptor or donor) exists if the interatomic distances are defined as follows: $d\left(X \ldots O_{W}\right)$ or $d\left(X \ldots H_{W}\right) \leq r_{\min }$ ( $r_{\min }$ is the first minimum of the corresponding RDF) and the donor-acceptor angle $\theta=\mathrm{Hw}-\mathrm{O}_{\mathrm{w}} \ldots \mathrm{X}$ (when water acts as HB donor) or $\theta=\mathrm{X}-\mathrm{Y} \ldots \mathrm{O}_{\mathrm{W}}$ ( $\mathrm{Y}$ is the Favipiravir atom connected to $\mathrm{X}$, when $\mathrm{X}$ is the donor atom) is such as $\theta \leq 30^{\circ} 37,38$ (note also that the symbol - corresponds to intramolecular vectors and the symbol ... to intermolecular ones). Using these geometric criteria, we focused on the $\mathrm{H}_{\mathrm{N}} \ldots \mathrm{O}_{\mathrm{w}}, \mathrm{O}_{\mathrm{C}} \ldots \mathrm{H}_{\mathrm{w}}, \mathrm{O}_{\mathrm{h}} \ldots \mathrm{H}_{\mathrm{w}}$ and $\mathrm{F} \ldots \mathrm{H}_{\mathrm{w}}$ hydrogen bonds and calculated the fractions of configurations where the Favipiravir molecule forms 0-2 of each type of bond. The so-obtained results are presented in Figure 3. From this Figure it can be observed that the fraction of the configurations where Favipiravir forms one and two $\mathrm{H}_{\mathrm{N}} \ldots \mathrm{O}_{\mathrm{w}}$ and $\mathrm{O}_{\mathrm{C}} \ldots \mathrm{H}_{\mathrm{w}}$ hydrogen bonds are higher in comparison with the other two types of hydrogen bonds $\left(\mathrm{O}_{h} \ldots \mathrm{H}_{\mathrm{W}}\right.$ and $\left.\mathrm{F} \ldots \mathrm{H}_{\mathrm{W}}\right)$. In the latter cases, the fraction of configurations with zero hydrogen bonds is much more pronounced. This is a clear indication that the $\mathrm{H}_{\mathrm{N}} \ldots \mathrm{O}_{\mathrm{W}}$ and $\mathrm{O}_{\mathrm{C}} \ldots \mathrm{H}_{\mathrm{W}}$ hydrogen bonds are the most pronounced in the system. This is also clearly reflected on the average number of hydrogen bonds of each type formed by the Favipiravir molecule. The average numbers $<\mathrm{n}$ нв $>$ per Favipiravir molecule for the investigated hydrogen bond types $\mathrm{H}_{\mathrm{N}} \ldots \mathrm{O}_{\mathrm{W}}, \mathrm{O}_{\mathrm{C}} \ldots \mathrm{H}_{\mathrm{W}}, \mathrm{O}_{\mathrm{h}} \ldots \mathrm{H}_{\mathrm{W}}$ and $\mathrm{F} \ldots \mathrm{H}_{\mathrm{W}}$ are $1.20,1.02,0.60$ and 0.29 , respectively. 


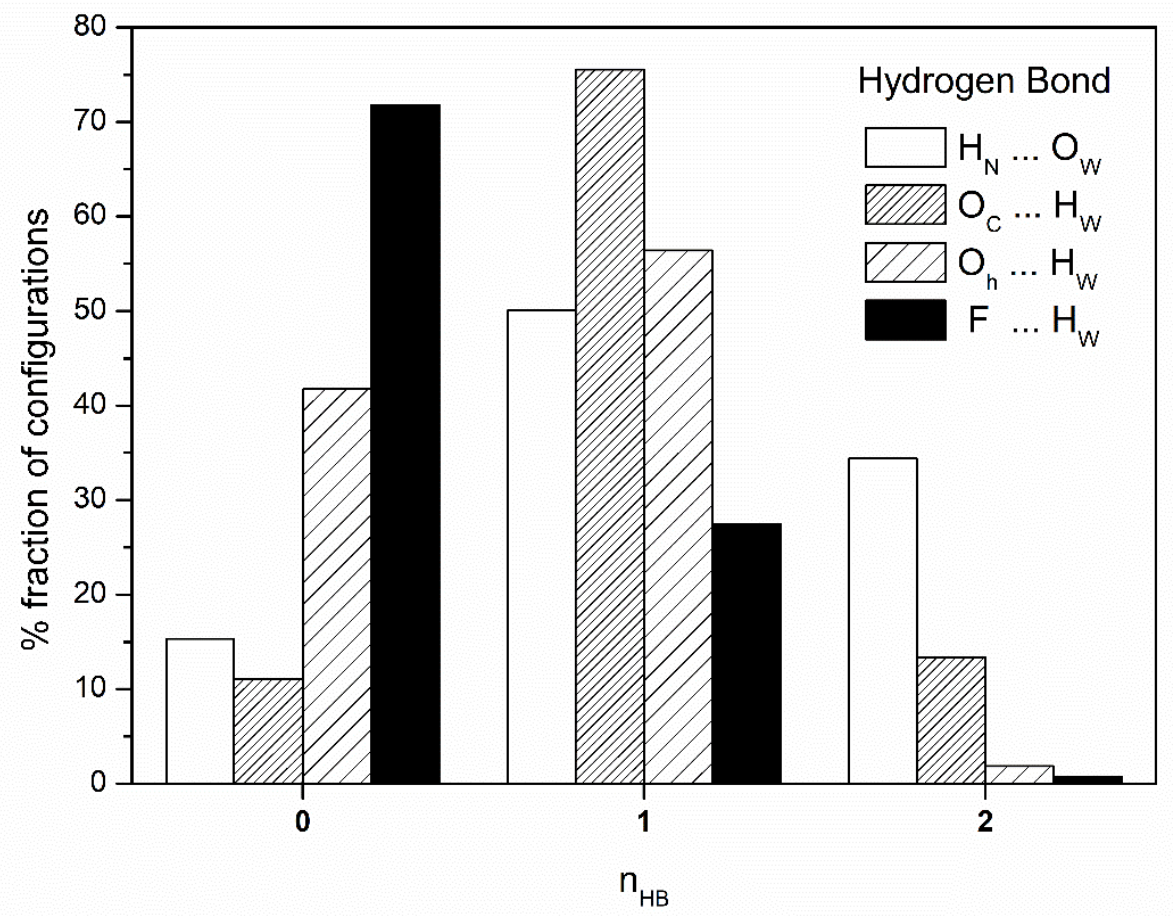

Figure 3: Calculated hydrogen bond statistics for the different types of Favipiravir-water hydrogen bonds.

Apart from the static description of the hydrogen bonds formed between Favipiravir and the water molecules, their dynamics were also investigated in terms of the corresponding $C_{H B}(t)$ time correlation functions (tcf) ${ }^{39-41}$ :

$$
C_{H B}(t)=\frac{\left\langle h_{i j}(0) \cdot h_{i j}(t)\right\rangle_{t^{*}}}{\left\langle h_{i j}(0)^{2}\right\rangle}
$$

while the HB lifetime $\tau_{\text {нв }}$ is defined as:

$$
\tau_{H B}=\int_{0}^{\infty} C_{H B}(t) \cdot d t
$$

The variable $h_{i j}$ is such as $h_{i j}(t)=1$ when molecule $\mathrm{j}$ is hydrogen bonded with molecule $\mathrm{i}$ at times 0 and $t$, and the corresponding hydrogen bond persists for a period longer than $\mathrm{t}^{*}$, 
otherwise, $h_{i j}(t)=0$. As in the case of residence dynamics, $t^{*}=0$ corresponds to the continuous HB dynamics, and $t^{*}=\infty$ corresponds to the intermittent one. The calculated continuous and intermittent tcfs $C_{H B}{ }^{C}(t)$ and $C_{H B}{ }^{I}(t)$ for all the investigated types of hydrogen bonds are presented in Figure 4.
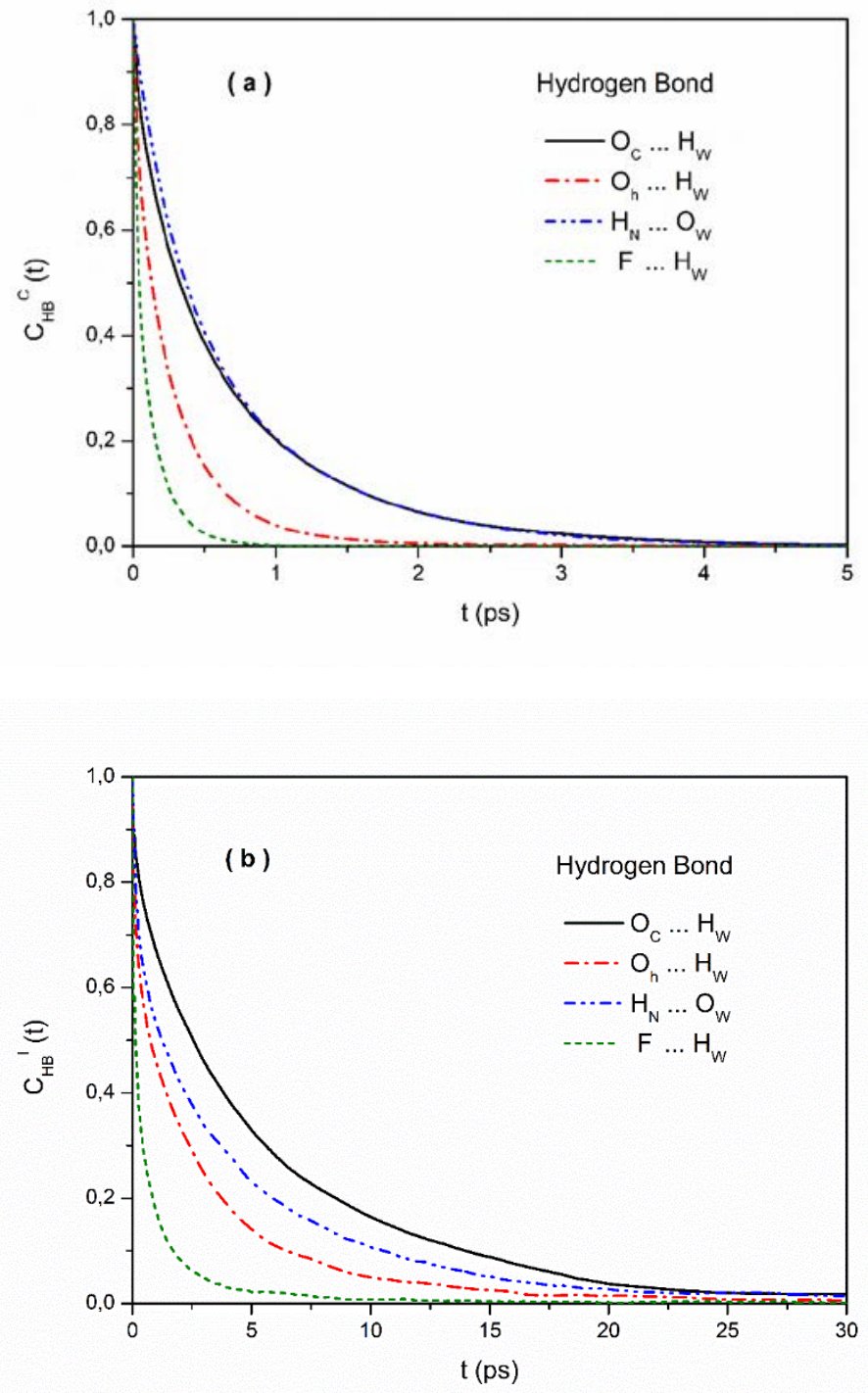

Figure 4: Calculated continuous and intermittent hydrogen bond time correlation functions for the different types of Favipiravir-water hydrogen bonds. 
The corresponding calculated continuous and intermittent HB lifetimes, $\tau_{H B}{ }^{C}$ and $\tau_{H B}{ }^{I}$ are also presented in Table 3. From these data it can be clearly seen that the trends regarding the intermittent HB lifetimes follow the sequence: $\tau_{H B}{ }^{I}\left(\mathrm{O}_{\mathrm{C}} \ldots \mathrm{H}_{\mathrm{W}}\right)>\tau_{H B}{ }^{I}\left(\mathrm{H}_{\mathrm{N}} \ldots \mathrm{O}_{\mathrm{W}}\right)>\tau_{H B}{ }^{I}\left(\mathrm{O}_{\mathrm{h}} \ldots \mathrm{H}_{\mathrm{W}}\right)>\tau_{H B}{ }^{I}(\mathrm{~F} \ldots \mathrm{HW})$. The continuous lifetimes of the $\mathrm{O}_{C} \ldots \mathrm{H}_{\mathrm{W}}$ and $\mathrm{H}_{\mathrm{N}} \ldots \mathrm{O}_{\mathrm{W}}$ hydrogen bonds are almost the same and longer than the continuous lifetimes of the $\mathrm{O}_{h} \ldots \mathrm{H}_{\mathrm{w}}$ and the $\mathrm{F} \ldots \mathrm{H}_{\mathrm{w}}$ hydrogen bonds, with the latter having a very short continuous lifetime $(0.1 \mathrm{ps})$. As we may therefore observe the lifetimes of the $\mathrm{O}_{\mathrm{C}} \ldots \mathrm{H}_{\mathrm{W}}$ and $\mathrm{H}_{\mathrm{N}} \ldots \mathrm{O}_{\mathrm{W}}$ hydrogen bonds are comparable with the lifetimes of water-water hydrogen bonds in pure bulk water, predicted in previous studies ${ }^{39-41}$, whereas in the cases of the $\mathrm{O}_{\mathrm{h}} \ldots \mathrm{H}_{\mathrm{w}}$ and, particularly, the $\mathrm{F} \ldots \mathrm{H}_{\mathrm{w}}$ hydrogen bonds, their lifetimes are much shorter. These findings clearly indicate that in general the interactions between Favipiravir and the water molecules are not particularly strong.

Table 3: Calculated average number of hydrogen bond types formed per Favipiravir molecule and their corresponding continuous and intermittent HB lifetimes.

\begin{tabular}{|c|c|c|c|c|}
\hline H. Bond Type & $\mathrm{O}_{\mathrm{C}} \ldots \mathrm{H}_{\mathrm{w}}$ & $\mathrm{H}_{\mathrm{N}} \ldots \mathrm{O}_{\mathrm{w}}$ & $\mathrm{O}_{\mathrm{h}} \ldots \mathrm{H}_{\mathrm{w}}$ & $\mathrm{F} \ldots \mathrm{H}_{\mathrm{W}}$ \\
\hline $\begin{array}{c}\left\langle n_{H B}\right\rangle \\
\text { (per Favipiravir } \\
\text { molecule) }\end{array}$ & 1.02 & 1.20 & 0.60 & 0.29 \\
\hline$\tau_{H B}{ }^{C}(\mathrm{ps})$ & 0.62 & 0.64 & 0.26 & 0.10 \\
\hline$\tau_{H B}{ }^{I}(\mathrm{ps})$ & 5.40 & 3.93 & 2.60 & 0.77 \\
\hline
\end{tabular}

As mentioned in the introduction, providing quantitative descriptions of the diffusion of drugs in aqueous solutions could be crucial for the understanding of the role of unstirred water layers on drug permeation in biological membranes. Therefore, in the present study we also calculated the self-diffusion coefficient of Favipiravir in water from the molecular translational mean square displacement (MSD) using the well-known Einstein relation:

$$
D_{S}=\frac{1}{6} \lim _{t \rightarrow \infty} \frac{1}{t}\left\langle\left|\vec{r}_{i}(0)-\vec{r}_{i}(t)\right|^{2}\right\rangle
$$


The calculated MSDs for Favipiravir and water are presented in Figure 5. The resulting self-diffusivities for Favipiravir and water are $D_{S}($ Favipravir $)=0.58 \cdot 10^{-9} \mathrm{~m}^{2} \cdot \mathrm{s}^{-1}$ and $D_{S}\left(H_{2} \mathrm{O}\right)=1.99 \cdot 10^{-9} \mathrm{~m}^{2} \cdot \mathrm{s}^{-1}$, respectively. As it can be clearly noticed, the self-diffusion coefficient of Favipiravir is more than three times lower in comparison with the value for water, this later value being similar to that obtained for bulk water ${ }^{42}\left(2.299 \cdot 10^{-9} \mathrm{~m}^{2} \cdot \mathrm{s}^{-1}\right)$. The calculated self-diffusion of Favipiravir in water has been presented in Table 4 together with the measured values of the self-diffusion of several representative pharmaceutical compounds in water, using several experimental techniques, which have already been presented in the literature ${ }^{9,43-46}$.

Table 4: Calculated translational self-diffusion coefficient of Favipiravir, presented together with previously reported experimental values of common representative antiinflammatory drugs, antibiotics and corticosteroids in aqueous solutions at very low concentration. In parentheses, the techniques used to evaluate the self-diffusion coefficient of each pharmaceutical compound are also mentioned.

\begin{tabular}{|c|c|}
\hline Compound & $\begin{array}{l}\text { D (in water) } \\
\left(10^{-9} \mathbf{m}^{2} / \mathrm{s}\right)\end{array}$ \\
\hline Favipiravir (This Work) & $0.58(\mathrm{MD})$ \\
\hline Aspirin & 0.65 (NMR) $^{41}$ \\
\hline Paracetamol & $\begin{array}{c}0.66 \text { (NMR) }^{41}, 0.66 \text { (Taylor Dispersion) }^{43} \text {, } \\
0.78 \text { (UV-Vis) }{ }^{9}\end{array}$ \\
\hline Salicylic acid & $0.76(\mathrm{NMR})^{41}$ \\
\hline 2-acetamidobenzoic acid & 0.58 (NMR) $^{41}$ \\
\hline Penicillin G & $\begin{array}{c}0.64 \text { (UV-Vis) } \\
0.40^{42} \\
\text { (open-ended capillary tube technique) }\end{array}$ \\
\hline Ampicillin & $\begin{array}{c}0.46^{42} \\
\text { (open-ended capillary tube technique) }\end{array}$ \\
\hline Tetracycline & $0.58(\mathrm{UV}-\mathrm{Vis})^{9}$ \\
\hline Trimethoprim & 0.56 (UV-Vis) $^{9}$ \\
\hline Ketoprofen & 0.64 (UV-Vis) $^{9}$ \\
\hline Beclomethasone & $\begin{array}{c}0.67 \\
\text { (chromatographic broadening method) }^{44}\end{array}$ \\
\hline Prednisone & $\begin{array}{c}0.73 \\
\text { (chromatographic broadening method) }^{44}\end{array}$ \\
\hline
\end{tabular}




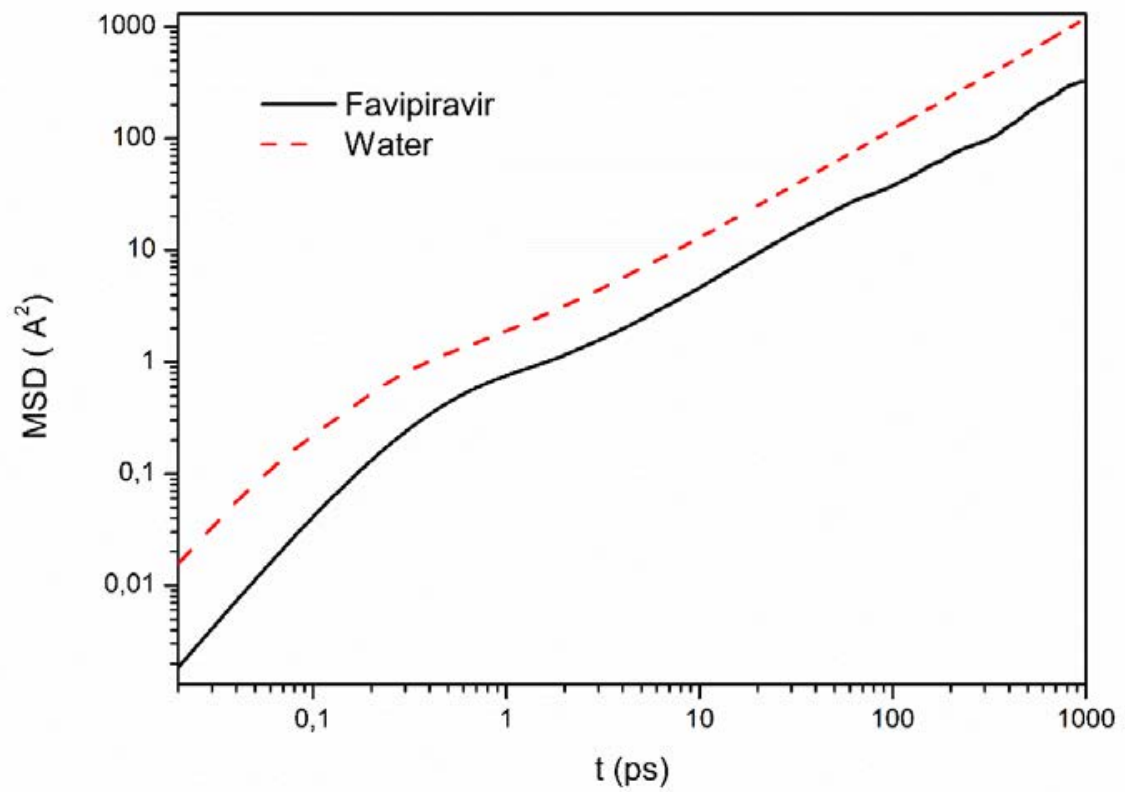

Figure 5: Calculated translational mean square displacements for Favipiravir and water.

Taking into account that in general reported self-diffusion values may depend on the experimental technique and the force fields employed in simulation studies, the main purpose of this comparison is to demonstrate that the diffusion of Favipiravir in water is in a similar range with the diffusion of common painkillers and anti-inflammatory drugs (e.g. paracetamol, aspirin, ketoprofen), as well as antibiotics (e.g. tetracycline, trimethoprim, Penicillin G) and corticosteroids for asthma treatment (e.g. beclomethasone, prednisone) in a very similar concentration range.

Apart from the translational dynamics, rotational dynamics could also significantly affect the mechanisms of drug permeability and might serve as probes of the size and symmetry dependence of the dynamics of molecules in confined environments, as it has been shown in our previous study ${ }^{47}$. Interestingly, another previous study in the literature has also shown that the rotation of the medium used for the drug transport can induce an accelerated drug release ${ }^{48}$. The study of rotational dynamics of nanoparticles on cell membranes can also lead to a better understanding of the mechanisms of drug delivery and further assist the rational design of surface modification strategies for drug delivery vectors under various circumstances ${ }^{49}$. Therefore, rotational dynamics can be very important from the 
study of pharmacokinetics, although they are often neglected and attention is primarily paid on the translational dynamics. In the case of solutes dissolved in water, the rotational dynamics is strongly affected by their local environment and particularly the HB network around them ${ }^{50}$. Translational diffusion of these solutes is often coupled to their rotational dynamics which in turn are coupled to similar motions of the surrounding water molecule ${ }^{50}$. The reorientational dynamics of the Favipiravir in the present study was also studied in terms of the Legendre reorientational time correlation functions for specific intramolecular vectors of the cation:

$$
C_{\ell R}(t)=P_{\ell}\left\langle\vec{u}_{i}(0) \cdot \overrightarrow{u_{i}}(t)\right\rangle
$$

The corresponding Legendre reorientational correlation times can be calculated using the following equation:

$$
\tau_{\ell R}=\int_{0}^{\infty} C_{\ell R}(t) \cdot d t
$$

In Eq. $4 \overrightarrow{u_{i}}$ is a unit intramolecular vector associated with a molecule $\mathrm{i}$, and $P_{\ell}$ is a Legendre polynomial of order $\ell$. The calculated first and second order Legendre reorientational time correlation functions for several representative intramolecular vectors of Favipiravir and the O-H vector of water molecules are presented in Figure 6 and the estimated corresponding correlation times in Table 5. From these figures, it can be seen that the calculated reorientational times for the different intramolecular vectors of Favipiravir are about 5-7 times longer in comparison with the values corresponding to the $\mathrm{O}-\mathrm{H}$ vector of the water molecules. This finding signifies that the rotational motions in Favipiravir are much slower in comparison with water, this trend being even more pronounced than for the translational dynamics mentioned above. The ratio of the calculated first and second order Legendre reorientational times $\tau_{1 R} / \tau_{2 R}$ for the investigated intramolecular vectors of Favipiravir is also in the range of 2.3-2.4, clearly indicating the deviation from the well-known equation $\tau_{1 R}=3 \cdot \tau_{2 R}$ which is valid only in the case of diffusive dynamics ${ }^{51-53}$. 

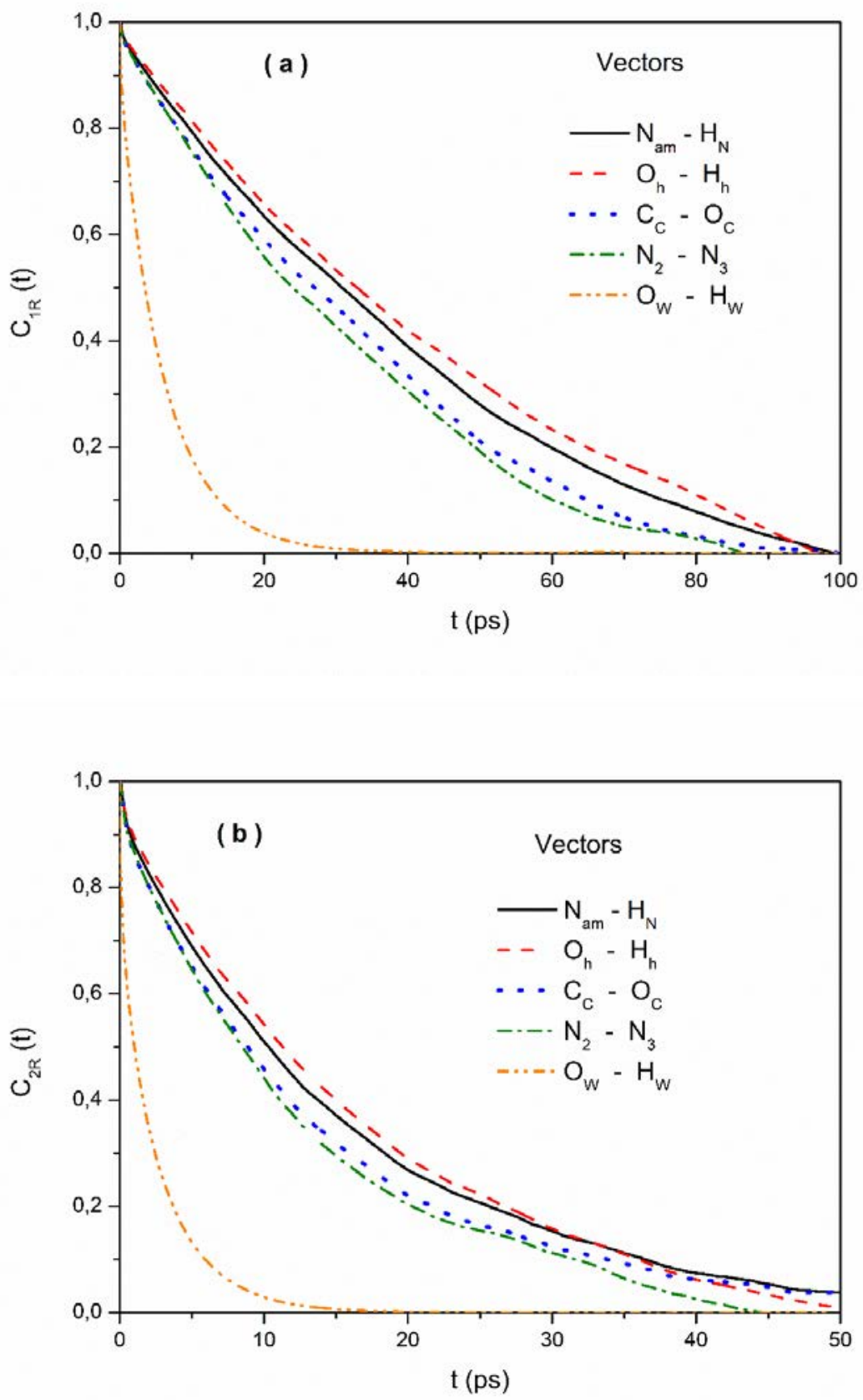

Figure 6: Calculated first and second order Legendre reorientational correlation functions for several representative intramolecular vectors of Favipiravir and the $\mathrm{O}-\mathrm{H}$ vector of water molecules. 
Table 5: Calculated first and second order Legendre reorientational correlation times for several representative intramolecular vectors of Favipiravir and the O-H vector of water molecules.

\begin{tabular}{|c|c|c|}
\hline Vector & $\boldsymbol{\tau}_{\mathbf{1 R}}(\mathbf{p s})$ & $\boldsymbol{\tau}_{\mathbf{2 R}}(\mathbf{p s})$ \\
\hline $\mathrm{N}_{\mathrm{am}}-\mathrm{H}_{\mathrm{N}}$ & 35.2 & 15.4 \\
\hline $\mathrm{O}_{\mathrm{h}}-\mathrm{H}_{\mathrm{h}}$ & 37.8 & 15.7 \\
\hline $\mathrm{C}_{\mathrm{C}}-\mathrm{O}_{\mathrm{C}}$ & 30.8 & 13.4 \\
\hline $\mathrm{N}_{2}-\mathrm{N}_{3}$ & 28.5 & 12.3 \\
\hline $\mathrm{O}_{\mathrm{W}}-\mathrm{H}_{\mathrm{W}}$ & 5.6 & 2.2 \\
\hline
\end{tabular}

\section{Conclusions}

In the present treatment, a combination of static quantum chemical calculations and classical molecular dynamics simulations has been employed to investigate the hydration structure and dynamics of the antiviral drug Favipiravir. In this framework a rigid all-atom force field for Favipiravir has been employed in the molecular dynamics simulations in order to reveal the local intermolecular structure around this antiviral drug molecule, as well as related dynamics. The so-obtained results demonstrated that the Favipiravir atoms which exhibit the strongest interactions with the water molecules are the hydrogen atoms $\mathrm{H}_{\mathrm{N}}$ of the $-\mathrm{NH}_{2}$ group of Favipiravir, the oxygen atom of the carbonyl group $\mathrm{O}_{\mathrm{C}}$ and the oxygen atom of the hydroxyl group $\mathrm{O}_{\mathrm{h}}$. These atoms form hydrogen bonds of the type $\mathrm{H}_{\mathrm{N}} \ldots \mathrm{O}_{\mathrm{W}}, \mathrm{O}_{\mathrm{C}} \ldots \mathrm{H}_{\mathrm{W}}, \mathrm{O}_{\mathrm{h}} \ldots \mathrm{H}_{\mathrm{W}}$ with the surrounding water molecules. Less prominent hydrogen bonds $\mathrm{F} \ldots \mathrm{H}_{\mathrm{W}}$, between the fluorine atom of Favipiravir and the hydrogen atoms of the water molecules have also been observed. The dynamics of these hydrogen bonds have been investigated and the trends regarding their intermittent HB lifetimes are $\tau_{H B}{ }^{I}\left(\mathrm{O}_{\mathrm{C}} \ldots \mathrm{H}_{\mathrm{W}}\right)>\tau_{H B}{ }^{I}\left(\mathrm{H}_{\mathrm{N}} \ldots \mathrm{O}_{\mathrm{W}}\right)>\tau_{H B}{ }^{I}\left(\mathrm{O}_{\mathrm{h}} \ldots \mathrm{H}_{\mathrm{W}}\right)>\tau_{H B}{ }^{I}\left(\mathrm{~F} \ldots \mathrm{H}_{\mathrm{W}}\right)$. The continuous lifetimes are in the sub picosecond range and intermittent lifetimes in the range of 0.8-5.4 ps. Providing quantitative descriptions of the diffusion of drugs in aqueous solutions could also be crucial for the understanding of the role of unstirred water layers on drug permeation in biological membranes. For this reason, the self-diffusion coefficient of Favipiravir in water was also calculated and was found to be comparable to the values measured for other common painkillers and anti-inflammatory drugs (e.g. paracetamol, 
aspirin, ketoprofen), as well as antibiotics (e.g. tetracycline, trimethoprim, Penicillin G) and corticosteroids for asthma treatment (beclomethasone, prednisone). It has also been found that it is about three times lower in comparison with the diffusion of the water molecules in the aqueous solution. The rotational motions of Favipiravir were also investigated and it was found that the calculated reorientational times for the different intramolecular vectors of Favipiravir are about 5-7 times larger in comparison with the values corresponding to the $\mathrm{O}-\mathrm{H}$ vector of the water molecules. This finding signifies that the rotational motions in Favipiravir are even more retarded in comparison with water than the translational ones. Furthermore, the evaluation of the first and second order Legendre reorientational times for selected intramolecular vectors of Favipiravir has also revealed that these dynamics are not diffusive.

The understanding of the hydration structure and diffusion mechanisms of active pharmaceutical ingredients in water could be correlated with their in vivo drug dissolution rate and could be used in a first-step screening approach to classify drugs based on their bioavailability. Furthermore, the training of force field for drug molecules with antiviral activity, such as Favipiravir, could be very useful in future in silico studies to understand in detail the molecular-scale phenomena which could help in targeting and blocking the infection pathways of several viruses, with particular emphasis on the SARS-CoV-2 coronavirus.

\section{Acknowledgements}

The use of the computational facilities at the Institute Charles Gerhardt of the University of Montpellier, the Computer Simulation in Condensed Matter Research Group (SIMCON) at the Department of Physics of the Technical University of Catalonia (UPC) and the Physical Chemistry Laboratory at the Chemistry Department of the National and Kapodistrian University of Athens are gratefully acknowledged. E. G. acknowledges financial support from the Ministerio de Ciencia, Innovación y Universidades of Spain, Grant PGC2018-099277-B-C21 (MCIU/AEI/ERDF). 


\section{References}

1) Bloom, D.E.; Cadarette, D. Infectious Disease Threats in the Twenty-First Century: Strengthening the Global Response. Front. Immunol. 2019, 10, 549.

2) Smith, K.M.; Machalaba, C.C.; Seifman, R.; Feferholtz, Y.; Karesh, W.B. Infectious disease and economics: The case for considering multi-sectoral impacts. One Health 2019, 7, 100080.

3) Chaudhuri, S.; Symons, J.A.; Deval, J. Innovation and trends in the development and approval of antiviral medicines: 1987-2017 and beyond. Antiviral Res. 2018, 155, 76-88.

4) Liu, C.; Zhou, Q.; Li, Y.; Garner, L.V.; Watkins, S.P.; Carter, L.J.; Smoot, J.; Gregg, A.C.; Daniels, A.D.; Jervey, S.; Albaiu, D. Research and Development on Therapeutic Agents and Vaccines for COVID-19 and Related Human Coronavirus Diseases. ACS Cent. Sci. 2020, 6, 315-331.

5) Li, G.; De Clercq, E. Therapeutic options for the 2019 novel coronavirus (2019-nCoV). Nat. Rev. Drug Discov. 2020, 19, 149-150.

6) Anderson; A.C. The process of structure based drug design. Chem. Biol. 2003, 10, 787797.

7) Brown; D.G., Shotton; E.J. Diamond: shedding light on structure-based drug discovery. Phil. Trans. R. Soc. A 2015, 373, 20140468.

8) Smith, M.; Smith, J.C. Repurposing Therapeutics for COVID-19: Supercomputer-Based Docking to the SARS-CoV-2 Viral Spike Protein and Viral Spike Protein-Human ACE2 Interface. 2020 ChemRxiv. Preprint. https://doi.org/10.26434/chemrxiv.11871402.v4

9) di Cagno, M.P.; Clarelli, F.; Våbenø, J.; Lesley, C.; Rahman, S.D.; Cauzzo, J.; Franceschinis, E.; Realdon, N.; Stein, P.C. Experimental Determination of Drug Diffusion Coefficients in Unstirred Aqueous Environments by Temporally Resolved Concentration Measurements. Mol. Pharmaceutics 2018, 15, 1488-1494. 
10) Zakeri-Milani, P.; Barzegar-Jalali, M.; Azimi, M.; Valizadeh, H. Biopharmaceutical classification of drugs using intrinsic dissolution rate (IDR) and rat intestinal permeability. Eur. J. Pharm. Biopharm. 2009, 73, 102-106.

11) Hayton, W.L. Rate-limiting barriers to intestinal drug absorption: a review. $J$ Pharmacokinet. Biopharm. 1980, 8, 321-334.

12) Chiou, W.L. Effect of 'Unstirred' water layer in the intestine on the rate and extent of absorption after oral administration. Biopharm. Drug Dispos. 1994, 15, 709-717.

13) Korjamo, T.; Heikkinen, A.T.; Mönkkönen, J. Analysis of Unstirred Water Layer in In Vitro Permeability Experiments. J. Pharm. Sci. 2009, 98, 4469-4479.

14) di Cagno, M.P.; Stein, P.C. Studying the effect of solubilizing agents on drug diffusion through the unstirred water layer (UWL) by localized spectroscopy. Eur. J. Pharm. Biopharm. 2019, 139, 205-212.

15) Frenkel, D.; Smit, B. Understanding Molecular Simulation: From Algorithms to Applications (2 ${ }^{\text {nd }}$ Edition), Academic Press, 2001.

16) Allen, M. P.; Tildesley, D. J. Computer Simulations of Liquids, Oxford University Press, Oxford, 1987.

17) Hossain, S.; Kabedev, A.; Parrow, A.; Bengström, C.A.S.; Larsson, P. Molecular simulation as a computational pharmaceutics tool to predict drug solubility, solubilization processes and partitioning. Eur. J. Pharm. Biopharm. 2019, 137, 46-55.

18) Zhu, Q.; Lu, Y.; He, X.; Liu, T.; Chen. H.; Wang, F.; Zheng, D.; Dong, H.; Ma, J. Entropy and Polarity Control the Partition and Transportation of Drug-like Molecules in Biological Membrane. Sci. Rep. 2017, 7, 17749.

19) Weiner, P.K.; Langridge, R.; Blaney, J.M.; Schaefer, R.; Kollman, P.A. Electrostatic potential molecular surfaces. Proc. Natl. Acad. Sci. USA 1982, 79, 3754-3758.

20) Furuta, Y.; Komeno, T.; Nakamura, T. Favipiravir (T-705), a broad spectrum inhibitor of viral RNA polymerase. Proc. Jpn. Acad. Ser. B 2017, 93, 449-463. 
21) Bocan, T.M.; Basuli, F.; Stafford, R.G.; Brown, J.L.; Zhang, X.; Duplantier, A.J.; Swenson, R.E. Synthesis of $\left[{ }^{18} \mathrm{~F}\right]$ Favipiravir and Biodistribution in $\mathrm{C} 3 \mathrm{H} / \mathrm{HeN}$ Mice as Assessed by Positron Emission Tomography. Sci. Rep. 2019, 9, 785.

22) Madelain, V.; Guedj, J.; Mentré, F.; Nguyen, T.H.T.; Jacquot, F.; Oestereich, L.; Kadota, T.; Yamada, K.; Taburet, A.-M.; de Lamballerie, X.; Raoul, H. Favipiravir Pharmacokinetics in Nonhuman Primates and Insights for Future Efficacy Studies of Hemorrhagic Fever Viruses. Antimicrob. Agents Chemother. 2016, 61, e01305-16.

23) Nguyen, T.H; Guedj, J.; Anglaret X.;, Laouenan, C.; Madelain, V.; Taburet, A.-M.; Baize, S.; Sissoko, D.; Pastorino, B.; Rodallec, A.; Piorkowski, G.; Carazo, S.; Conde, M.N.; Gala, J.L.; Bore, J.A.; Carbonnelle, C.; Jacquot, F.; Raoul, H.; Malvy, D.; de Lamballerie, X.; Mentré, F. Favipiravir pharmacokinetics in Ebola-Infected patients of the JIKI trial reveals concentrations lower than targeted. PLoS Negl. Trop. Dis. 2017, 11, e0005389.

24) Gaussian 09, Revision D.01, M. J. Frisch, G. W. Trucks, H. B. Schlegel, G. E. Scuseria, M. A. Robb, J. R. Cheeseman, G. Scalmani, V. Barone, G. A. Petersson, H. Nakatsuji, X. Li, M. Caricato, A. Marenich, J. Bloino, B. G. Janesko, R. Gomperts, B. Mennucci, H. P. Hratchian, J. V. Ortiz, A. F. Izmaylov, J. L. Sonnenberg, D. Williams-Young, F. Ding, F. Lipparini, F. Egidi, J. Goings, B. Peng, A. Petrone, T. Henderson, D. Ranasinghe, V. G. Zakrzewski, J. Gao, N. Rega, G. Zheng, W. Liang, M. Hada, M. Ehara, K. Toyota, R. Fukuda, J. Hasegawa, M. Ishida, T. Nakajima, Y. Honda, O. Kitao, H. Nakai, T. Vreven, K. Throssell, J. A. Montgomery, Jr., J. E. Peralta, F. Ogliaro, M. Bearpark, J. J. Heyd, E. Brothers, K. N. Kudin, V. N. Staroverov, T. Keith, R. Kobayashi, J. Normand, K. Raghavachari, A. Rendell, J. C. Burant, S. S. Iyengar, J. Tomasi, M. Cossi, J. M. Millam, M. Klene, C. Adamo, R. Cammi, J. W. Ochterski, R. L. Martin, K. Morokuma, O. Farkas, J. B. Foresman, and D. J. Fox, Gaussian, Inc., Wallingford CT, 2016.

25) Dunning, T.H. Jr Gaussian basis sets for use in correlated molecular calculations. I. The atoms boron through neon and hydrogen. J. Chem. Phys. 1989, 90, 1007-1023.

26) Shi, F.; Li, Z.; Kong, L.; Xie, Y.; Zhang, T.; Xu, W. Synthesis and crystal structure of 6-fluoro-3-hydroxypyrazine-2-carboxamide. Drug. Discov. Ther. 2014, 8, 117-120. 
27) Rhyman, L.; Tursun, M.; Abdallah, H.H.; Choong, Y.S.; Parlak, C.; Kharkar, P.; Ramasami, P. Theoretical investigation of the derivatives of favipiravir (T-705) as potential drugs for Ebola virus. Phys. Sci. Rev. 2018, 3, 20170198.

28) Breneman, C.M.; Wiberg, K.B. Determining Atom-Centered Monopoles from Molecular Electrostatic Potentials. The Need for High Sampling Density in Formamide Conformational Analysis. J. Comp. Chem. 1990, 11, 361-373.

29) Jorgensen, W.L.; Maxwell, D.S.; Tirado-Rives, J. Development and Testing of the OPLS All-Atom Force Field on Conformational Energetics and Properties of Organic Liquids. J. Am. Chem. Soc. 1996, 118, 11225-11236.

30) Onufriev, A.V.; Izadi, S. Water models for biomolecular simulations. WIREs Comput. Mol. Sci. 2018, 8, e1347. doi: 10.1002/wcms.1347.

31) Onufriev, A.V.; Izadi, S. Accuracy limit of rigid 3-point water models. J. Chem. Phys. 2016, 145, 074501.

32) Abascal, J.L.F.; Vega, C. A general purpose model for the condensed phases of water: TIP4P/2005. J. Chem. Phys. 2005, 123, 234505.

33) Martínez, L.; Andrade, R.; Birgin, E. G.; Martínez, J. M. PACKMOL: a package for building initial configurations for molecular dynamics simulations, J. Comput. Chem. 2009, 30, 2157-2164.

34) Hoover, W. G. Canonical dynamics: Equilibrium phase-space distributions. Phys. Rev. A 1985, 31, 1695-1697.

35) Hoover, W. G. Constant-pressure equations of motion. Phys. Rev. A 1986, 34, 24992500.

36) Smith, W.; Forester, T. R. DL_POLY_2.0: A General-Purpose Parallel Molecular Dynamics Simulation Package. J. Mol. Graphics 1996, 14, 136-141.

37) Luzar, A.; Chandler, D. Hydrogen-bond kinetics in liquid water. Nature 1996, 379, 5557. 
38) Skarmoutsos, I.; Guardia, E.; Samios, J. Local structural fluctuations, hydrogen bonding and structural transitions in supercritical water. J Supercrit. Fluids 2017, 130, 156164.

39) Guardia, E.; Laria, D.; Marti, J. Hydrogen Bond Structure and Dynamics in Aqueous Electrolytes at Ambient and Supercritical Conditions. J. Phys. Chem. B 2006, 110, 63326338.

40) Rapaport, D. C. Hydrogen bonds in water. Mol. Phys. 1983, 50, 1151-1162.

41) Guardia, E.; Skarmoutsos, I.; Masia, M. Hydrogen Bonding and Related Properties in Liquid Water: A Car-Parrinello Molecular Dynamics Simulation Study. J. Phys. Chem. B 2015, 119, 8926-8938.

42) Holz, M.; Heil. S.R.; Sacco, A. Temperature-dependent self-diffusion coefficients of water and six selected molecular liquids for calibration in accurate ${ }^{1} \mathrm{H}$ NMR PFG measurements. Phys. Chem. Chem. Phys. 2000, 2, 4740-4742.

43) Blokhina, S.V.; Volkova, T.V.; Golubev, V.A.; Perlovich, G.L. Understanding of Relationship between Phospholipid Membrane Permeability and Self-Diffusion Coefficients of Some Drugs and Biologically Active Compounds in Model Solvents. Mol. Pharmaceutics 2017, 14, 3381-3390.

44) Padfield, J.M.; Kellaway, I.W. The diffusion of penicillin G and ampicillin through phospholipid sols. J. Pharm. Pharmac. 1975, 27, 348-352.

45) Ribeiro, A.C.F.; Barros, M.C.F.; Veríssimo, L.M.P.; Santos, C.I.A.V.; Cabral, A.M.T.D.P.V.; Gaspar, G.D.; Esteso, M.A. Diffusion coefficients of paracetamol in aqueous solutions. J. Chem. Thermodynamics 2012, 54, 97-99.

46) Seki, T.; Mochida, J.; Okamoto, M.; Hosoya, O.; Juni, K.; Morimoto, K. Measurement of Diffusion Coefficients of Parabens and Steroids in Water and 1-Octanol. Chem. Pharm. Bull. 2003, 51, 734-736.

47) Skarmoutsos, I.; Eddaoudi, M.; Maurin, G. Peculiar Molecular Shape and Size Dependence of the Dynamics of Fluids Confined in a Small-Pore Metal-Organic Framework. J. Phys. Chem. Lett. 2018, 9, 3014-3020. 
48) Mitsumata, T.; Kakiuchi, Y.; Takimoto, J.-I. Fast Drug Release Using Rotational Motion of Magnetic Gel Beads. Adv. Phys. Chem. 2008, 671642. https://doi.org/10.1155/2008/671642

49) Gu, Y.; Sun, W.; Wang, G.; Fang, N.J. Single Particle Orientation and Rotation Tracking Discloses Distinctive Rotational Dynamics of Drug Delivery Vectors on Live Cell Membranes. J. Am. Chem. Soc. 2011, 133, 5720-5723.

50) Banerjee, P.; Bagchi, B. Ions’ motion in water. J. Chem. Phys. 2019, 150, 190901.

51) Debye, P. Polar Molecules; Dover Publications: New York, 1929.

52) Laage, D.; Hynes, J. T. On the Molecular Mechanism of Water Reorientation. J. Phys. Chem. B 2008, 112, 14230-14242.

53) Skarmoutsos, I.; Spyrogiannopoulos, L.; Kainourgiakis, E.; Samios, J. On the interplay between the local structure and dynamics in low concentration mixtures of $\mathrm{H}_{2} \mathrm{O}$ and HOD in the $\left[\mathrm{Emim}^{+}\right]\left[\mathrm{TF} 2 \mathrm{~N}^{-}\right]$room temperature ionic liquid. J. Mol. Liq. 2019, 289, 111135.

\section{TOC Figure}

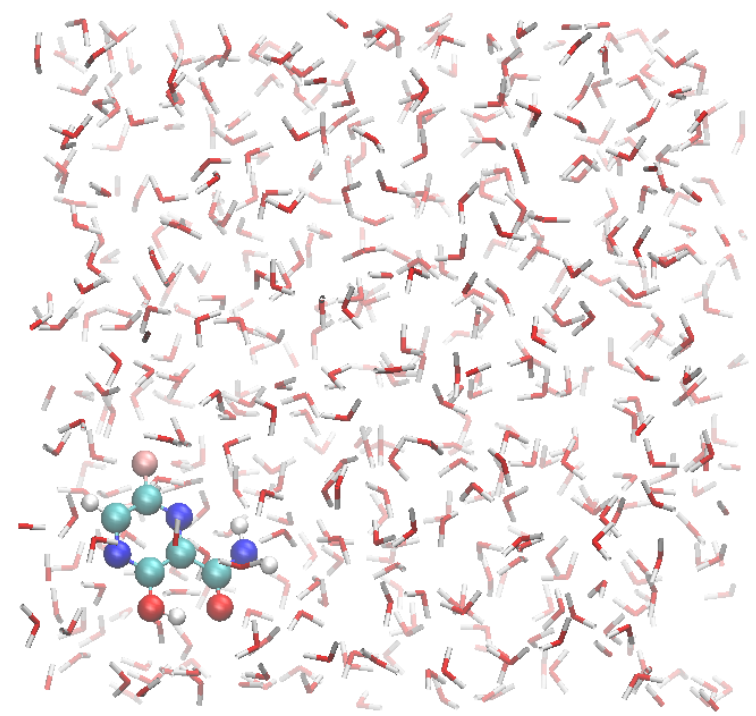

